\title{
The Design and Application of Mobile Media System
}

\author{
Changqing Bai $^{1}$ Min Ju$^{1}$ Yao Wei ${ }^{1}$ \\ ${ }^{1}$ Beijing University of Posts and Telecommunications, Beijing, China
}

\begin{abstract}
The Mobile Media System is proposed in the article, it relies on Fixed-line broadband, 3G/GPRS, wireless LAN communication network, which can conduct remote configuration and unified management for the different types of mobile media terminal, implementing timing, orientation, scheduled content publishing of the information. In addition, according to the system, a Mobile Media Interactive System based on GIS is designed, it can release preferential information overlay on the GIS data of many merchants. Through the Near Field Communication (NFC), the Mobile Terminal can download them. The system seeks to enhance the user of both businesses and consumers experiences.
\end{abstract}

Keywords: Mobile Media System; GIS; Mobile Media Terminal; NFC

\section{The Design of Mobile Media System}

The Mobile Media System (short for MMS) relies on Fixed-line broadband, 3G/GPRS, wireless LAN communication network, which can conduct remote configuration and unified management for the different types of mobile media terminal, implementing timing, orientation, scheduled content publishing of the information, it can not only meet the needs of various industries, for instance, dissemination of information, operations of advertising, but also provide an effective means for rapid deployment and efficient operation of the new media.

The system architecture is shown in Fig. 1. The system consists of four parts: Media Display Center Management Platform (referred to as MDCMP), Mobile Media Terminal (MMT), Network Access (NA) and Media Release Status Data Needs Department (MRSDND).

Network Access has wireless and cable. Wireless access includes 3G / HSDPA, GPRS and Wi-Fi. Cable access includes ADSL and Ethernet.

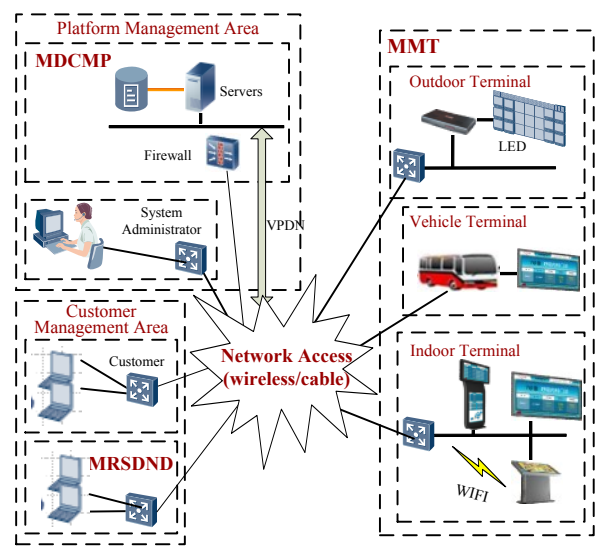

Fig. 1: The chart of MMS architecture.

\subsection{The framework of MDCMP}

MDCMP is the core part of the MMS. It is responsible for layout, review and release of media material, for processing the security alarm, and for managing, monitoring, counting and maintaining the media release data and terminal state. Its logic framework chart is shown in Fig. 2. 
The basic data of Intranet Integration Platform (IIP), including the following types of database: Basic data, Real-time data, Operational reports and Historical data.

The layer of Application Services Support Platform (ASSP), including the following types of service: Communication Distribution Service (CDS), Media Release Service (MRS), Automatic Upgrade Service (AUS), Data Exchange Service (DES) and Industry Databases Service (IDS).

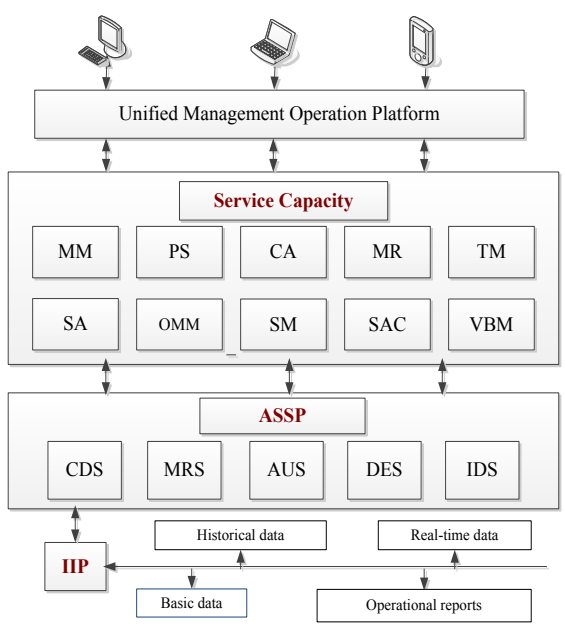

Fig. 2: The chart of MDCMP framework.

The layer of Service Capacity, including the following types of ability: Material Management (MM), Program Scheduling (PS), Content Approval (CA), Media Release (MR), Terminal Management (TM), Statistical Analysis (SA), Operation and Maintenance Management (OMM), Security Management (SM), System Administration \&Control (SAC) and Value-added Business Management (VBM).

\subsection{The structure of MMT}

The terminal of MMS (referred to as Mobile Media Terminal, MMT for short) be- longs to one of the Internet of Things terminals. MMT is the front-end equipment of the MMS, it is able to receive real-time playlists and multimedia material pushed by MDCMP, to be responsible for storing and updating the multimedia materials, to arrange broadcast according to playlists. Meanwhile, through the network, MDCMP can remotely perform some operations such as query, monitoring, diagnostic, configuration and control on the Media Terminals.

MMT was laid in a public place to provide users with information services. According to their usage scene, the Mobile Media Terminal can be divided into three types: Vehicle media terminal, Indoor media terminal, Outdoor media terminal.

The structure of MMT can be divided into four modules: Interface Modules (IM), Master Module (MM), Communication Module (CM) and Power Module (PM), as shown in Fig. 3.

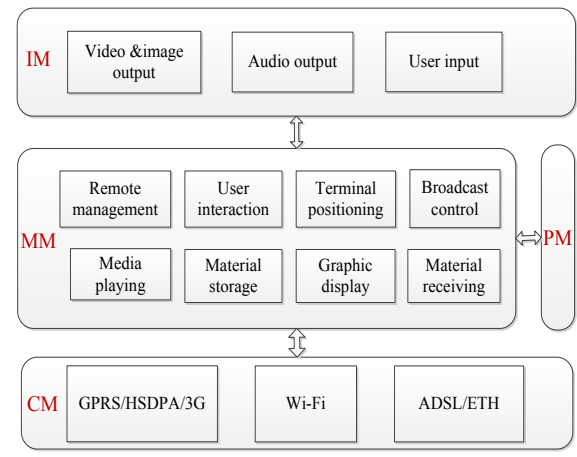

Fig. 3: The chart of MMT structure.

IM implements user interface, including the output of images, videos and audio, as well as the input of users.

MM implements the functions of control and management for Media release business, mainly including the necessary functions such as material receiving, material storage, media playing, graphic display, remote management, software up- 
grades, and the optional functions such as user interaction, terminal positioning.

$\mathrm{CM}$ provides one or more wireless/cable access to make the MMT connected to the Internet or Private Network, and also provides the channel for data transmission between Media Terminal and MDCMP or the third-party platform.

PM is responsible for power supply and energy management.

\section{The Application of MMS}

With the development of Mobile Internet and Terminal technology, some merchants have begun to use mobile terminals such as mobile phones to distribute electronic coupons to consumers, in pursuit of higher transmission efficiency and rate of return on advertising investment.

Currently, the methods of distributing electronic coupons mainly have the following three questions:

Too extensive; not locate the potential consumers precisely; and abusing the information makes consumer feel disgusted

Consumers need to find preferential information by themselves; the range of selection is relatively small and blindness

Terminals only provide coupons of surrounding merchants in specific locations; while the crowd is large, consumers need to queue for printing, user experience is worse; high costs

In order to solve the above-mentioned limitations, based on Mobile Media System, a GIS-based Interactive Mobile Media System (referred to as GIMMS) is proposed in the paper.

\subsection{The Overview of GIMMS}

In the GIMMS, the MMT can provide advertising preferential information shown on the map to consumers more accurately. In the meantime, the NFC terminal of consumers can download the preferential information by interacting with the mobile media terminal through the near-field communications technology.

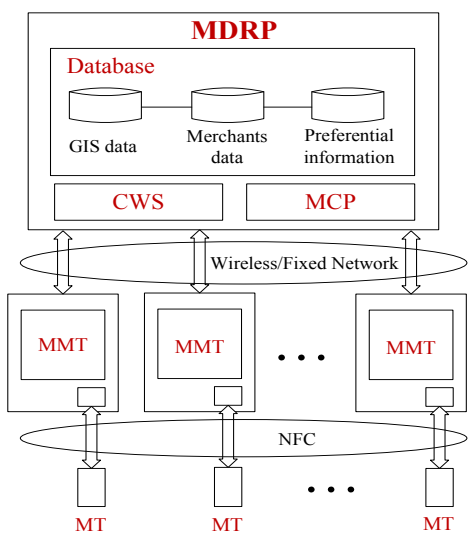

Fig. 4: The chart of GIMMS.

As shown in Fig. 4, the system consists of three parts: Mobile Media Release Platform (MDRP), Mobile Media Terminals (MMT) and Mobile Terminals (MT).

MDRP contains Database, Client Web Server (CWS) and Management Control Platform (MCP). The Database records three data layers: GIS data, Merchant data superimposed on the GIS data and the corresponding preferential information; The Client Web Server receives GIS data and the Merchant data, and then sends them to Mobile Media Terminal; The Management Control Platform is used to manage scheduling of data communication among Mobile Media Terminals.Mobile Media Terminals are applied to interact with consumers and Mobile Terminals. It includes interactive screen and Near Field Communication (NFC) module. Through the Mobile Media Terminal, GIS data and Merchants data will be presented to consumers in graphical form. According to their own preferences, consumers select merchants to view the preferential information. After choosing the desired preferential information, Mo- 
bile Media Terminal will load the information into NFC tags.

Mobile Terminal is the ultimate carrier of the preferential information. It has built-in NFC module. When the NFC Mobile Terminals induce NFC tags embedded in the Mobile Media Terminal, the terminal will read the information on the tags which rendered in the form of SMS or MMS.

\section{Summary}

Based on the above-described Mobile Media System, the paper proposes a GISbased interactive mobile media system and method. The system and method can help the existing merchants solve some limitations of distributing advertising preferential information, for example behaviors extensive, low accuracy and abuse of information.

The system can sent coupons to potential consumers in a more precise way. In addition, it is capable of providing quick access to help consumers get more referrals while obtaining preferential information, achieving the purpose that "accurate delivery" and "effective reach". It is not only to save human and material, but also to enhance the user experience of businesses and consumers, the marketing ef- fect is better than that of traditional advertising and leaflets mode.

\section{Acknowledgements}

This work is supported by the Researches on Comprehensive Measurement Verification Technology for Climate Observation\#210280.

\section{References}

[1] "A distributed mobile multimedia operating system," Proceedings of the Seventh IASTED International Conference on Internet and Multimedia Systems and Applications, pp. 44-49, 2003.

[2] "Media distribution system," Nippon Telegraph and Telephone Corp., Volume: 3, Issue: 8, pp. 32-34, 2005.

[3] "A System of Secure Virtual Coupons Using NFC Technology," Proc. Of the Fifth Annual IEEE International Conference on Pervasive Computing and Communications Workshops, 2007.

[4] "Geographical bias on social media and geo-local contents system with mobile devices," Hawaii International Conference on System Sciences, 2012. 